\author{
„WIR BRAUCHEN EIN KLARES BEKENNTNIS ZUR \\ INNOVATION UND INVESTITION IN ENABLING \\ TECHNOLOGIES, UM SO DIE CHANCEN DER \\ BIOTECHNOLOGIE AM STANDORT DEUTSCHLAND \\ AUCH IN DER ZUKUNFT OPTIMAL NUTZEN ZU \\ KÖNNEN.“
}

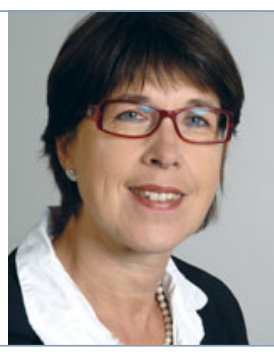

Dorothee Ambrosius

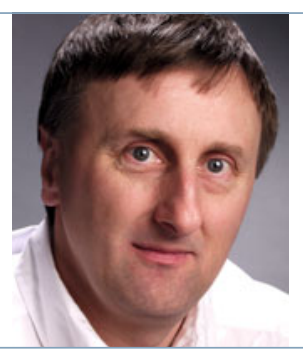

Johannes Buchner

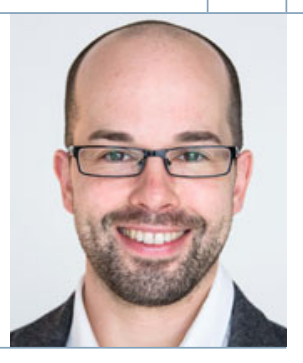

Matthias J. Feige

\title{
Wir brauchen eine next generation protein science in Deutschland!
}

DOI: $10.1007 / \mathrm{s} 12268-017-0863-8$

(C) Springer-Verlag 2017

Die rekombinante Herstellung von Proteintherapeutika ist eine Schlüsseltechnologie mit außerordentlichem Innovationspotenzial. So wurde mit humanem Insulin das erste rekombinante Produkt aus Escherichia coli 1982 zugelassen und hat bis heute Insulin tierischen Ursprungs vollständig ersetzt, bei gleichbleibend hoher Qualität im Tonnenmaßstab. In den 1970er Jahren entwickelten die Nobelpreisträger César Milstein und Georges J. F. Köhler die Technologie zur Herstellung monoklonaler Antikörper, die heute mit bis zu zehn Zulassungen pro Jahr die weltweit umsatzstärkste Klasse aller Biotherapeutika darstellen. Rekombinante Antikörper haben zu neuen Ansätzen und wesentlichen Verbesserungen in der Therapie schwerer Krankheiten wie Krebs und Rheuma beigetragen.

Die rasante Entwicklung und erfolgreiche Anwendung von biotherapeutischen Arzneimitteln belegen eindrucksvoll, dass diese ein Behandlungsprinzip ermöglichen, das für klassische chemische Arzneimittel nicht zugänglich ist. Dabei hängt der medizinische Erfolg der Biotherapeutika sowohl von den Innovationen in der Biotechnologie als auch von enabling technologies $\mathrm{ab}$, die zur Entwicklung und Herstellung dieser komplexen Biomoleküle notwendig sind. Eine enabling technology war zu Beginn die Molekularbiologie, welche die rekombinante Expression und gerichtete Mutagenese erlaubte und sich heute vor allem im Bereich der Zellbiologie rasant weiterentwickelt (CRISPR). Danach verlagerte sich der Schwerpunkt auf die Protein-bezogenen Techniken, wie die chromatographische Reinigung und Proteinanalytik. Viele der bahnbrechenden methodischen Entwicklungen liegen aber bereits Jahrzehnte zurück, Verbesserungen sind häufig nur noch inkrementeller Natur. War Methodenentwicklung früher Voraussetzung für Forschung, so ist sie nun eher eine Nischenaktivität. Es gibt seitens der universitären Forschung keinen großen Anreiz zur Innovation auf diesem Gebiet, und die Ausbildung einer neuen Generation von Wissenschaftlern findet zunehmend Kit-basiert statt, teilweise unter Vernachlässigung des tiefer gehenden Verständnisses der zugrunde liegenden Prozesse. Andererseits sind durch den Erfolg der Bio- therapeutika und die neue Ära der Biosimilars die Anforderungen bezüglich eines ganzheitlichen Verständnisses des Herstellungsprozesses und dessen Einfluss auf die Produktqualität rapide gestiegen. Andere Bereiche, wie beispielsweise die zielgerichtete Veränderung von Zuckerstrukturen auf Proteinen zur Beeinflussung von Stabilität und Wirkmechanismus, stecken noch in den Kinderschuhen.

Das traditionelle Verhältnis von Entwickler und Anwender hat sich umgekehrt: An Forschungsinstituten werden vielfach Standardmethoden angewandt, in der Industrie kommen aufgrund des enorm gestiegenen Bedarfs an Biotherapeutika und sensitiver diagnostischer Assays high-end-Methoden und -Technologien zum Einsatz - und werden dort auch weiterentwickelt. Allerdings fokussiert die Industrie ihre neuen technologischen und methodischen Konzepte darauf, aktuelle Produktentwicklungen zu unterstützen. Bahnbrechende Innovationen haben es so schwer, in den etablierten Prozessen und kurzen Zyklen Aufnahme zu finden.

Die Erforschung von neuen enabling technologies wird in Deutschland derzeit weder in der Industrie noch an den akademischen Instituten genügend vorangetrieben. Im internationalen Vergleich gefährdet diese Innovationslücke die Zukunftssicherung einer Schlüsseltechnologie, die neben der akademischen Exzellenz ein wichtiger Wirtschaftsmotor für den Standort Deutschland darstellt.

Im angelsächsischen Raum findet Technologieentwicklung oft an der Schnittstelle zwischen Universität und Industrie in Start-ups statt - und wird gezielt durch incubators an den Universitäten gefördert. Diese Schnittstelle ist gerade in der Biotechnologie, mit wenigen Ausnahmen auf nationaler und EU-Ebene, noch zu schwach ausgeprägt. Auch Karrieren zwischen Industrie und Forschungsinstituten sind in den USA üblich, in Deutschland die Ausnahme.

Wenn momentan auch noch die akademische Forschung nur als user der Technologien funktioniert, so wird dies mittelfristig ihr Innovationspotenzial limitieren, da die technologische Weiterentwicklung und der Zugang zu neuen wissenschaftlichen Erkenntnissen stets Hand in Hand gehen.

Für den Erhalt und weiteren Aufbau einer konkurrenzfähigen Biotechnologie in Deutsch- land benötigen wir deshalb eine aktive Forschungsförderung mit dezidierten Förderinstrumenten für Technologieentwicklung. Die Interaktion zwischen Industrie und Universität ist hierfür essenziell, um gemeinsame Interessen zu eruieren, aber auch, um den state of the art und Engpässe zu definieren. Darüber hinaus sind die Transferaufgabe der Industrie und ihre Bereitschaft zur Implementierung neuer Methoden und Herstellprozesse für biotherapeutische Arzneimittel im Dialog mit den Behörden essenziell.

Eine wesentliche Voraussetzung ist weiterhin die profunde wissenschaftliche und technische Ausbildung des Nachwuchses an Instituten mit internationaler Reputation, die das vernetzte Denken der Lebenswissenschaften im Dialog mit anderen Disziplinen weiterentwickeln. Wir brauchen ein klares Bekenntnis zur Innovation und Investition in die enabling technologies, um so die Chancen der Biotechnologie am Standort Deutschland auch in der Zukunft optimal nutzen zu können.

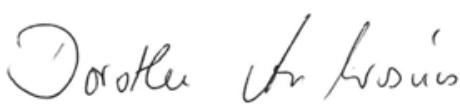

Dr. Dorothee Ambrosius,

Head of CMC Strategy Biologicals, Boehringer Ingelheim Biberach
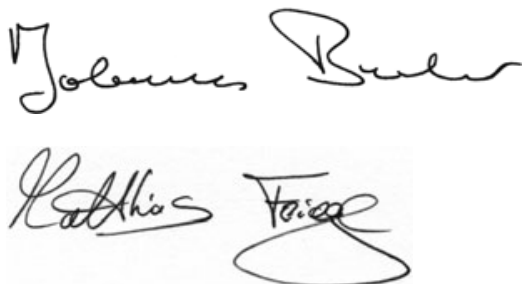

Prof. Johannes Buchner,

Prof. Matthias J. Feige,

Department Chemie, TUMünchen

Korrespondenzadresse:

Dr. Dorothee Ambrosius

Boehringer Ingelheim Pharma GmbH \& Co. KG

Birkendorfer Straße 65

D-88397 Biberach

Tel.: 07351-54-4830

dorothee.ambrosius@boehringer-ingelheim.com 